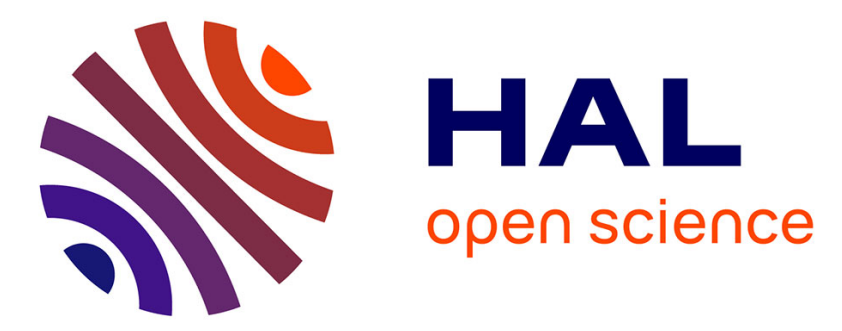

\title{
Three-dimensional simulation of aluminium extrusion by the $\alpha$-shape based natural element method
}

Icíar Alfaro, David Bel, Elías Cueto, Manuel Doblaré, Francisco Chinesta

\section{To cite this version:}

Icíar Alfaro, David Bel, Elías Cueto, Manuel Doblaré, Francisco Chinesta. Three-dimensional simulation of aluminium extrusion by the $\alpha$-shape based natural element method. Computer Methods in Applied Mechanics and Engineering, 2006, 195 (33-36), pp.4269-4286. 10.1016/j.cma.2005.08.006 . hal-01007384

\section{HAL Id: hal-01007384 \\ https://hal.science/hal-01007384}

Submitted on 17 Jan 2017

HAL is a multi-disciplinary open access archive for the deposit and dissemination of scientific research documents, whether they are published or not. The documents may come from teaching and research institutions in France or abroad, or from public or private research centers.
L'archive ouverte pluridisciplinaire HAL, est destinée au dépôt et à la diffusion de documents scientifiques de niveau recherche, publiés ou non, émanant des établissements d'enseignement et de recherche français ou étrangers, des laboratoires publics ou privés. 


\title{
Three-dimensional simulation of aluminium extrusion by the $\alpha$-shape based natural element method
}

\author{
I. Alfaro ${ }^{\text {a }}$, D. Bel ${ }^{\text {a }}$, E. Cueto ${ }^{\text {a }}$, M. Doblaré ${ }^{\text {a }}$, F. Chinesta ${ }^{\text {b }}$ \\ ${ }^{\text {a }}$ Group of Structural Mechanics and Material Modelling (GEMM), Aragón Institute of Engineering Research (I3A), University of Zaragoza, \\ Betancourt Building, María de Luna, 5, E-50018 Zaragoza, Spain \\ b LMSP UMR 8106 CNRS ENSAM-ESEM, 151 Bvd. de l'Hôpital, F-75013 Paris, France
}

In this work we study the possible advantages of an updated Lagrangian perspective in the numerical simulation of aluminium extrusion. For this purpose, we have employed a well established meshless technique known as Natural Element Method (NEM). This technique presents some advantages over finite element simulations, such as no remeshing requirements and the accuracy of the approximation even with highly distorted triangles or tetrahedra. It is employed in conjunction with the notion of $\alpha$-shapes of the cloud of nodes in order to extract the geometry of the extrudate as it evolves. This state-of-the-art geometrical concept allows us to avoid complex geometrical checks of self-contact on the boundary of the domain. Aluminium is modelled as a rigid-plastic material, governed by a Sellars-Tegart-type law. The paper includes some examples that illustrate the potential of the method.

Keywords: Aluminium extrusion; Meshless; Meshfree; Natural element method; $\alpha$-shapes

\section{Introduction}

Direct aluminium extrusion is one of the most extended forming processes worldwide. Roughly speaking, it is a process used to produce long profiles by pressing a billet of hot aluminium through a hole with a certain shape. A schematic representation of the process is given in Fig. 1.

The design and manufacturing of extrusion dies has been traditionally driven by the experience of designers. Nowadays, numerical simulation tools - mainly based on finite elements - greatly simplify the design process, though no definitive automatization has been achieved. This is in part due to the complexity of the process (that involves large transformations in a coupled thermo-mechanical setting) and the limitations inherent to the FEM. These limitations are often due to the large mesh distortion during the simulated process. To avoid these, one of the possible solutions is to perform remeshing when required. But this method is far from being automatized in three dimensions. Other possibility is to employ Arbitrary Lagrangian-Eulerian simulations [20], in which the mesh displacement can be set independently of the material displacement, thus minimizing the remeshing process.

In this paper we turn back to an updated Lagrangian framework. Since the irruption of meshless or meshfree methods in the middle nineties $[4,11,17]$ a new possibility has opened. These methods are of different nature, but all share the same main characteristic: accuracy is not strongly affected by the nodal distribution. In addition, nodal connectivity is con- 


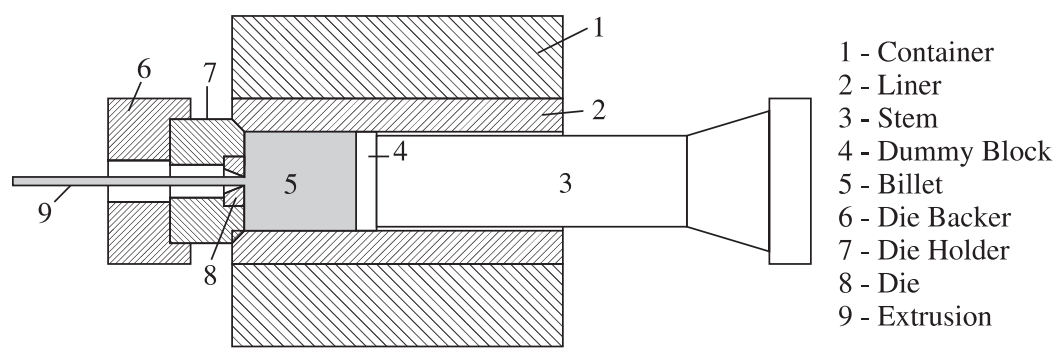

Fig. 1. Schematic representation of the extrusion process.

structed in a process transparent to the user, thus greatly alleviating the user effort in the pre-processing stage of the model. This allows us to establish updated Lagrangian models that do not suffer from mesh distortion.

Nevertheless, these methods have some drawbacks. Among these, imposition of essential boundary conditions is not straightforward, being necessary to employ some techniques like Lagrange multipliers, modified variational principles or others [17]. Other problems, like numerical integration errors, have been recently overcome or, at least, alleviated [6].

The natural element method [26,9] is a meshless method with somewhat different characteristics. Firstly, it is based on some kind of natural neighbour-based interpolation $[24,16]$. These interpolation schemes are strictly interpolant and, under some weak restrictions, they are strictly interpolant on the boundaries of the domain [8,7]. As other meshless methods, the employ of this kind of interpolation in a Galerkin framework has proved to render accurate results independently of the "mesh" distortion, as shown in [25,21].

In order to avoid complex geometrical checks in the free-surface flow of the extrudate, we have modelled the flow through a collection of three-dimensional $\alpha$-shapes [12] that evolve in time. This allows us to deal with a set of nodes only, without any explicit information on the geometry of the boundary. Thus, development of holes in the material or merge of flows are treated in a consistent manner with great accuracy in mass conservation.

In this paper we do not pursue the development of a novel model to describe aluminium behaviour, but to analyse the potential of use of $\alpha$-shape-based natural element methods to simulate complex forming processes like aluminium extrusion. In this paper we have modelled the hot aluminium as a viscoplastic material. This assumption has been accepted in a vast number of prior works, provided that plastic strains are large enough when compared to elastic ones. This has been often referred to as flow formulation $[33,32]$ in the forming processes community. Of course, this simple model does not allow us to predict "spring-back" phenomena. Some basic assumptions have also been taken regarding the simulation of extrudate-die contact, etcetera.

The outline of the paper is as follows. In Section 2 we introduce a brief review on aluminium behaviour during the extrusion process. In Section 3 we review the basics of natural neighbour interpolation and the natural element method. In Section 4 we describe the implementation of the coupled thermo-mechanical procedure and, finally, in Section 5 we present some results that show the potential of the method. The paper finishes summarising the most important conclusions in Section 6 .

\section{Aluminium behaviour during the extrusion process}

Extrusion, as briefly presented in the introduction before, is a forming process that causes large strains in the aluminium so as to be able to flow through the die, thus acquiring the desired shape. In this situation, strains are so large when compared to elastic strains, that it has been a common practice in many prior works (see $[33,31,32,20]$, just to cite some of the first and more recent works using this assumption) to neglect these and to assimilate the aluminium to a visco-plastic material. This assumption simplifies the numerical simulation, but that makes impossible the spring-back prediction, whose modelling requires more accurate behaviour laws also including elasticity. In this setting, aluminium can be considered as a particular instance of non-newtonian fluids, whose behaviour could be described by

$$
\dot{\boldsymbol{\varepsilon}}=f(\boldsymbol{\sigma}) \text {. }
$$

In the pioneer work by Zienkiewicz and Godbolet (see [31] and references therein), the visco-plastic strain rate tensor (symmetric part of the gradient of velocities) was defined by a general expression of the type

$$
\boldsymbol{d}^{\mathrm{vp}}=\dot{\gamma} \frac{\partial Y(\boldsymbol{\sigma}, \boldsymbol{q})}{\partial \boldsymbol{\sigma}},
$$

where $Y$ is the viscoplastic potential—usually coincident with the plastic criterion as has been considered here, $\dot{\gamma}$ is a scalar function given by 


$$
\dot{\gamma}=\frac{\langle g(Y(\boldsymbol{\sigma}, \boldsymbol{q}))\rangle}{\eta} \quad \text { with } \quad\langle x\rangle=\frac{x+|x|}{2},
$$

$\langle g\rangle$ is a monotone function that takes zero value only if $Y(\boldsymbol{\sigma}, \boldsymbol{q}) \leqslant 0, \eta$ is a positive parameter often called viscosity and $\boldsymbol{q}$ represent the hardening parameters. In what follows we will avoid the use of the vp superscript to indicate viscoplastic if there is no risk of confusion.

It is common to assume a von Mises type flow for the aluminium in such conditions, so as to give

$$
Y(\boldsymbol{\sigma}, \boldsymbol{q})=\bar{\sigma}(\boldsymbol{s})-\sigma_{\mathrm{y}}(\bar{d}, T),
$$

where $\boldsymbol{s}=\boldsymbol{\sigma}-\frac{\operatorname{tr} \sigma}{3} \boldsymbol{I}=\boldsymbol{\sigma}-p \boldsymbol{I}$ is the deviatoric part of the stress tensor, $\bar{\sigma}=\sqrt{\frac{3}{2} \boldsymbol{s}: \boldsymbol{s}}=\sqrt{3 J_{2}}$ represents the effective stress, and the yield stress $\sigma_{\mathrm{y}}$ follows a Sellars-Tegart law:

$$
\sigma_{\mathrm{y}}(\bar{d})=S_{m} \operatorname{arcsinh}\left[\left[\left(\frac{\bar{d}}{A}\right) \mathrm{e}^{Q / R T}\right]^{1 / m}\right],
$$

$S_{m}, m$ and $A$ are material parameters. $A$ is a factor depending on the magnesium and silicium matrix solute content (see [20] and references therein). $Q$ represents the activation energy of the deformation process, $R$ is the universal gas constant and, finally, $T$ is the absolute temperature. $\bar{d}$ is the only internal variable in this model and is sometimes called effective strain rate:

$$
\bar{d}=\sqrt{\frac{2}{3} \boldsymbol{d}: \boldsymbol{d}}
$$

Note that if the strain rate tends to zero, so does yield stress. This is not related to the well-known behaviour of aluminium, so it is a common practice [19] to modify the Sellars-Tegart law to include this initial strain rate, $\bar{d}_{0}$ :

$$
\sigma_{\mathrm{y}}(\bar{d})=S_{m} \operatorname{arcsinh}\left[\left[\left(\frac{\bar{d}_{1}}{A}\right) \mathrm{e}^{Q / R T}\right]^{1 / m}\right] \text { with } \bar{d}_{1}=\max \left\{\bar{d}, \bar{d}_{0}\right\} .
$$

If we combine now the general form of the strain rate tensor given in Eq. (2), assuming $\Psi=f(\boldsymbol{\sigma}, \boldsymbol{q})$, with Eq. (4), we arrive to

$$
\boldsymbol{d}=\dot{\gamma} \frac{3 \boldsymbol{s}}{2 \bar{\sigma}} .
$$

It is immediate now, by combining Eq. (6) and the definition of effective stress, $\bar{\sigma}$, to prove that $\dot{\gamma}$ is precisely the effective strain rate:

$$
\bar{d}=\frac{\dot{\gamma}}{\bar{\sigma}} \sqrt{\frac{3}{2} s: s}=\dot{\gamma}
$$

On the other hand, and by following the Perzyna-like model employed in Eqs. (2) and (3) and taking $g(f)=f$, we arrive to a relationship between equivalent stress and equivalent strain rate:

$$
\bar{d}=\dot{\gamma}=\frac{\left\langle\bar{\sigma}-\sigma_{\mathrm{y}}\right\rangle}{\eta} \Rightarrow \bar{\sigma}=\eta \bar{d}+\sigma_{\mathrm{y}} \quad \text { if } \bar{\sigma} \geqslant \sigma_{\mathrm{y}}
$$

that, introduced in Eq. (8), accounting Eq. (9), gives the following visco-plastic constitutive equation:

$$
\boldsymbol{s}=2 \frac{\eta \bar{d}+\sigma_{\mathrm{y}}(\bar{d})}{3 \bar{d}} \boldsymbol{d}
$$

Note that, depending on the $\eta$ value, return to the yield surface is done with different velocity.

Since it is common to describe aluminium behaviour as rigid-plastic (rather than viscoplastic) we employ null viscosity, so as to enforce $Y=\bar{\sigma}-\sigma_{\mathrm{y}}=0$, leading to

$$
\boldsymbol{s}=\frac{2 \sigma_{\mathrm{y}}}{3 \bar{d}} \boldsymbol{d} .
$$

Finally, the constitutive equation, accounting the incompressibility of plastic flow results:

$$
\boldsymbol{\sigma}=2 \mu \boldsymbol{d}-p \boldsymbol{I}, \quad \text { with } \mu=\frac{\sigma_{\mathrm{y}}}{3 \bar{d}} .
$$

Of course, this simple model has important limitations. Undoubtedly, the lack of elastic behaviour is one of the most important. Thus, spring-back cannot be predicted. However, as mentioned before, it has rendered good results and seems to be widely accepted in the forming processes community $[31,33,20]$. 


\section{The natural element method}

The natural element method (also known as natural neighbour Galerkin method) can be considered a member of the vast family of meshless methods. It relies on any of the various natural neighbour-based interpolation schemes to construct the space of trail and test functions of the Galerkin method. So, it is necessary to introduce these kind of interpolation prior to describe the characteristics of the NEM.

\subsection{Natural neighbour interpolation}

There exist various types of natural neighbour-based interpolations, but they all are based on the construction of the Delaunay triangulation [10] of the cloud of points, $\mathscr{D}$, used to discretise the domain. The Delaunay triangulation of a set of points $N=\left\{n_{1}, n_{2}, \ldots, n_{N}\right\}$ is the unique triangulation of the set that verifies the so-called empty circumcircle criterion. This means that no point of the set lies in the interior of a circle that passes through the three vertices of each triangle, see Fig. 2.

The dual structure of the Delaunay triangulation is the Voronoi diagram of the cloud [28]. For a given node $n_{I}$, its associated Voronoi cell is composed by all of the points which are closer to the node $n_{I}$ than to any other node. Formally,

$$
T_{I}=\left\{\boldsymbol{x} \in \mathbb{R}^{n}: d\left(\boldsymbol{x}, \boldsymbol{x}_{I}\right)<d\left(\boldsymbol{x}, \boldsymbol{x}_{J}\right) \forall J \neq I\right\},
$$

where $T_{I}$ is the Voronoi cell and $d(\cdot, \cdot)$ represents the Euclidean distance. In the problems considered in this paper, $n=3$. Two nodes whose Voronoi cells share one edge are called natural neighbours and hence the name of these interpolation schemes.

The first, and most obvious, interpolation scheme based on natural neighbours is the so-called nearest neighbour or Thiessen interpolation [27]. If we give the nodal value to the whole associated Voronoi cell, we obtain a $\mathscr{C}^{-1}$ interpolation scheme. This interpolation scheme is not suitable for solving second-order partial differential equations, but has been employed in [15] to construct mixed velocity-pressure approximations for the simulation of incompressible media (see Fig. 3).

The most extended form of natural neighbour-based interpolation is due to Sibson [24]. For the definition of Sibson interpolation it is necessary to previously introduce the concept of second-order Voronoi cell. It is defined as the locus of the points that have the node $n_{I}$ as the closest node and the node $n_{J}$ as the second closest node:

$$
T_{I J}=\left\{\boldsymbol{x} \in \mathbb{R}^{n}: d\left(\boldsymbol{x}, \boldsymbol{x}_{I}\right)<d\left(\boldsymbol{x}, \boldsymbol{x}_{J}\right)<d\left(\boldsymbol{x}, \boldsymbol{x}_{K}\right) \forall K \neq J ; \forall K \neq I\right\} .
$$

If a new point is added to a given cloud of points, the Voronoi cells will be modified by its presence. Sibson [23] defined the natural neighbour coordinates of a point $\boldsymbol{x}$ with respect to one of its neighbours $I$ as the ratio of the cell $T_{I}$ that is transferred to $T_{x}$ to the initial cloud of points to the total area of $T_{x}$. In other words, being $\kappa(x)$ and $\kappa_{I}(\boldsymbol{x})$ the Lebesgue measures of $T_{x}$ and $T_{x I}$ respectively, the natural neighbour coordinates of $\boldsymbol{x}$ with respect to the node $I$ is defined as

$$
\phi_{I}^{\mathrm{sib}}(\boldsymbol{x})=\frac{\kappa_{I}(\boldsymbol{x})}{\kappa(\boldsymbol{x})} .
$$

The resultant shape function depends obviously on the relative position of the nodes. An example for a node surrounded by other six is depicted in Fig. 4.

The resultant shape function has some remarkable properties (see [25] or [9] for more in-deep explanations and rigorous proofs of this behaviour). Firstly, it is smooth $\left(\mathscr{C}^{1}\right)$ everywhere except from the nodes, as can be seen from Fig. 4 . Natural neighbour (Sibson) shape functions posses linear completeness [25] and form a partition of unity. Therefore, it is possible to enrich natural neighbour interpolants in order to increase the order of the polynomial reproduced by the interpolation, as proposed in [3].

Recently, Hiyoshi and Sugihara [16] have generalised the form of natural neighbour interpolants. One different type of interpolation has attracted the interest of researchers, since it is slightly faster to compute, although gives less smooth interpolations. It has received the name of Laplace interpolant. The Laplace interpolant is defined by using geometrical entities

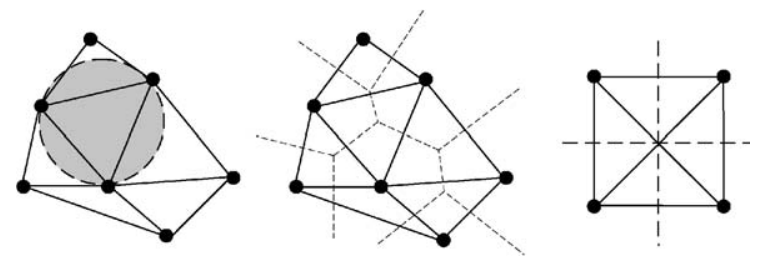

Fig. 2. Delaunay triangulation and Voronoi diagram of a cloud of points. On the right, an example of a degenerate distribution of nodes, with the two possible triangulations depicted. In this last case, four points lie in the same circumcircle and thus no single triangulation exists. 


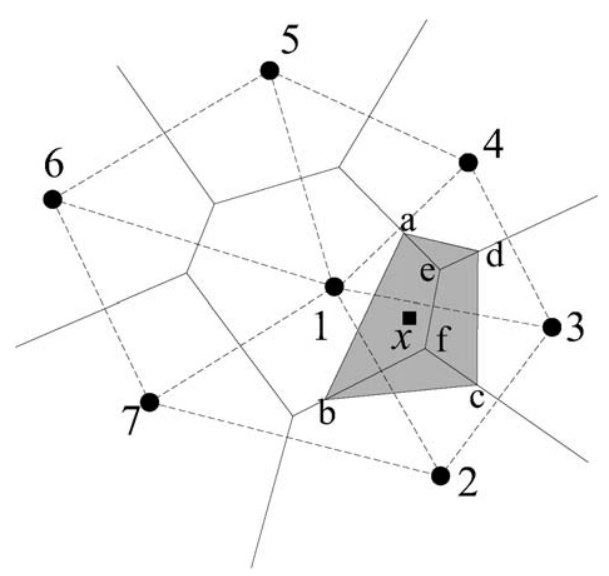

Fig. 3. Definition of the natural neighbour coordinates of a point $\boldsymbol{x}$.

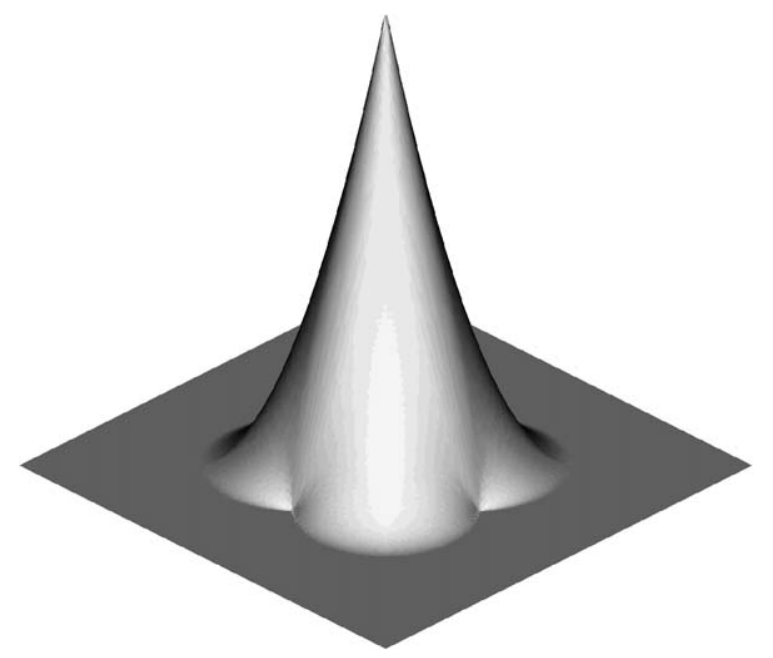

Fig. 4. Natural element (Sibson) shape function (photo courtesy N. Sukumar).

of one dimension less than the original space under consideration. If we define the cell intersection $t_{I J}=\left\{\boldsymbol{x} \in T_{I} \cap T_{J}\right.$, $J \neq I\}$ (note that $t_{I J}$ may be an empty set) we can define the value

$$
\alpha_{J}(\boldsymbol{x})=\frac{\left|t_{I J}\right|}{d\left(\boldsymbol{x}, \boldsymbol{x}_{J}\right)} \text {. }
$$

Thus, the point $\boldsymbol{x}$ shape function value with respect to node 4 in Fig. 5 is defined as

$$
\phi_{4}^{n s}(\boldsymbol{x})=\frac{\alpha_{4}(\boldsymbol{x})}{\sum_{J=1}^{n} \alpha_{J}(\boldsymbol{x})}=\frac{s_{4}(\boldsymbol{x}) / h_{4}(\boldsymbol{x})}{\sum_{J=1}^{n}\left[s_{J}(\boldsymbol{x}) / h_{J}(\boldsymbol{x})\right]},
$$

where $s_{J}$ represent the length of the Voronoi segment associated to node $J$ and $n$ represents the number of natural neighbours of the point under consideration, $\boldsymbol{x}$.

Derivatives of the Laplace shape function are not defined along the edges of the Delaunay triangles that lie inside its support (see [26]). For the purposes of the work here presented, Sibson's interpolation has been considered.

In the context of two- and three-dimensional approximations, the unknown variable is approximated in the form:

$$
\boldsymbol{u}^{h}(\boldsymbol{x})=\sum_{I=1}^{n} \phi_{I}(\boldsymbol{x}) \boldsymbol{u}_{I},
$$

where $\boldsymbol{u}_{I}$ is the vector of nodal displacements or velocities and $n$ the number of natural neighbours of each point $\boldsymbol{x}$. This leads to a $\mathscr{C}^{0}$ interpolation scheme. With the unknown variable thus approximated, a Galerkin discrete system of equations is then built. 


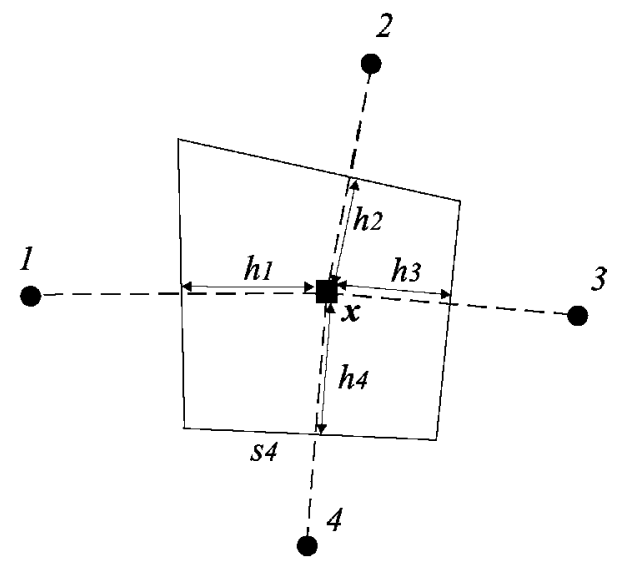

Fig. 5. Definition of non-Sibsonian coordinates.

\subsection{Properties of natural neighbour interpolation}

Firstly, unlike many other approximation techniques used in meshless methods, Sibson's interpolation scheme is strictly interpolant, i.e., the approximated surface pass through the data. This can be expressed as

$$
\phi_{I}\left(\boldsymbol{x}_{J}\right)=\delta_{I J}
$$

with $\delta_{I J}$ the Kronecker delta tensor.

The natural neighbour (Sibson) interpolant also has linear consistency. This can be demonstrated from the before mentioned partition of unity property and its ability to exactly reproduce linear fields (also known as local coordinate property, see [24]):

$$
\sum_{I=1}^{n} \phi_{I}(\boldsymbol{x}) \boldsymbol{x}_{I}=\boldsymbol{x} .
$$

The fact that Sibson functions form a partition of unity has been used in [15] to enrich the velocity field so as to give a mixed velocity-pressure approximation that verifies the inf-sup or Babuška-Brezzi condition.

One important property that derives from the before mentioned ones is that the imposition of essential boundary conditions in NE methods is straightforward. Like in the FEM, it is sufficient to prescribe nodal values to reproduce essential boundary conditions. In addition, natural neighbour interpolants can be strictly interpolant on the boundaries (convex or not), under very weak conditions, as demonstrated in [8] by using the theory of $\alpha$-shapes. This proof was later generalised in [7]. This means that no interior point of the domain takes influence on the boundary. A brief review of the theory of $\alpha$ shapes is presented in Section 4.

These properties are in sharp contrast with many of the most extended meshless methods, which usually need Lagrange multipliers, modified variational principles, penalty formulations or other methods to verify - often only at a node-wise level - essential boundary conditions (see [13] for an interesting review on this topic). This and other properties (like its sound geometrical properties, that will be further analysed next) make the NEM, in the authors' opinion, an appealing choice for the simulations of forming processes, and in particular, extrusion processes.

\section{Numerical implementation}

In this section we describe the numerical model of the extrusion process, as explained before, in the context of a natural neighbour Galerkin method. We firstly review the governing equations and the mixed approximation used to deal with the incompressibility conditions. Then, we describe the technique used to deal with the free-surface flow that extrusion processes involve.

\subsection{Governing equations}

We consider the balance of momentum equations, without inertia and mass terms

$$
\nabla \cdot \boldsymbol{\sigma}=\mathbf{0}
$$


and the assumed incompressibility of a von Mises-like flow:

$$
\nabla \cdot \boldsymbol{v}=0
$$

where $v$ represents the velocity field. The stress-strain rate relationship is given by Eq. (12). Parameters governing the aluminium yield stress (see Eq. (8)) are summarised in Table 1.

This rigid-plastic material is coupled with the following heat transfer equations:

$$
\nabla(k \nabla T)+\dot{r}-\left(\rho c_{p} \dot{T}\right)=0,
$$

where $k$ denotes the thermal conductivity, $\dot{r}$ the heat generation rate, $\rho$ the specific density and $c_{p}$ the specific heat of the metal. The rate of heat generation in the aluminium billet due to plastic deformation is given by

$$
\dot{r}=\beta \boldsymbol{\sigma}: \boldsymbol{d},
$$

where $\beta$ represents the fraction of mechanical energy transformed to heat and is assumed to be 0.9 [30]. Together with these equations, appropriate boundary conditions are considered:

$$
\begin{aligned}
& \boldsymbol{\sigma} \cdot \boldsymbol{n}=\overline{\boldsymbol{t}} \text { in } \Gamma_{\mathrm{t}}, \\
& \boldsymbol{v}=\overline{\boldsymbol{v}} \text { in } \Gamma_{\mathrm{v}},
\end{aligned}
$$

where $\Gamma_{\mathrm{t}}$ and $\Gamma_{\mathrm{v}}$ represent, respectively, the part of the boundary $\Gamma=\partial \Omega$ where tractions and velocities are prescribed. In addition, along the boundary, either temperature or heat flux is prescribed.

If we write the incremental variational equation at time $t+\Delta t$ we arrive to:

$$
\int_{\Omega(t+\Delta t)}\left(-\left(p^{t}+\Delta p\right) \boldsymbol{I}+2 \mu\left(\boldsymbol{d}^{t}+\Delta \boldsymbol{d}\right)\left(\boldsymbol{d}^{t}+\Delta \boldsymbol{d}\right)\right): \boldsymbol{d}^{*} \mathrm{~d} \Omega=0 .
$$

Due to the non-linear character of the constitutive equations, an iterative approach has been applied, using the NewtonRaphson scheme, thus leading to

$$
\begin{gathered}
\int_{\Omega(t+\Delta t)}\left(-\Delta \Delta p \boldsymbol{I}+2 \mu\left(\frac{\partial \mu\left(\boldsymbol{d}_{k}^{t+\Delta t}\right)}{\partial \boldsymbol{d}}: \Delta \Delta \boldsymbol{d}\right) \boldsymbol{d}_{k}^{t+\Delta t}+2 \mu\left(\boldsymbol{d}_{k}^{t+\Delta t}\right) \Delta \Delta \boldsymbol{d}\right): \boldsymbol{d}^{*} \mathrm{~d} \Omega \\
=-\int_{\Omega(t+\Delta t)}\left(-p_{k}^{t+\Delta t} \boldsymbol{I}+2 \mu\left(\boldsymbol{d}_{k}^{t+\Delta t}\right) \boldsymbol{d}_{k}^{t+\Delta t}\right): \boldsymbol{d}^{*} \mathrm{~d} \Omega,
\end{gathered}
$$

where the subscript $k$ indicates the iteration within a time increment. The incremental form of the incompressibility condition results

$$
\int_{\Omega(t+\Delta t)} \nabla \cdot(\Delta \Delta v) p^{*} \mathrm{~d} \Omega=-\int_{\Omega(t+\Delta t)} \nabla \cdot\left(\boldsymbol{v}_{k}^{t+\Delta t}\right) p^{*} \mathrm{~d} \Omega .
$$

If we approximate the velocities and pressures, as well as their variations, by employing a finite-dimensional set of basis functions, we arrive to a discrete form of the previous equations (Bubnov-Galerkin method)

$$
\begin{aligned}
& \Delta \Delta \boldsymbol{v}^{h}(\boldsymbol{x})=\sum_{I=1}^{n} \phi_{I}(\boldsymbol{x}) \Delta \Delta \boldsymbol{v}_{I}, \\
& \Delta \Delta p^{h}(\boldsymbol{x})=\sum_{I=1}^{n} \psi_{I}(\boldsymbol{x}) \Delta \Delta p_{I},
\end{aligned}
$$

where $n$ represents the number of nodes considered in the approximation. The functions $\psi_{I}(\boldsymbol{x})$ and $\phi_{I}(\boldsymbol{x})$ in this work represent some form of natural neighbour interpolation, which will be studied in the following section. This leads to the linear system:

Table 1

Material parameters for AA6063 aluminium alloy (values taken from [20])

\begin{tabular}{lll}
\hline Parameter & Units & Value \\
\hline $\bar{d}_{0}$ & $\mathrm{~s}^{-1}$ & 0.005 \\
$S_{m}$ & $\mathrm{MPa}$ & 25 \\
$m$ & & 5.4 \\
$A$ & $\mathrm{~s}^{-1}$ & $6 \times 10^{9}$ \\
$Q$ & $\frac{\mathrm{J}}{\mathrm{mol}}$ & $1.4 \times 10^{5}$ \\
$R$ & $\frac{\mathrm{J}}{\mathrm{mol} \mathrm{K}}$ & 8.314 \\
\hline
\end{tabular}




$$
\left.\begin{array}{cc}
\boldsymbol{K}_{k}^{t+\Delta t} & \boldsymbol{G} \\
\boldsymbol{G}^{T} & \mathbf{0}
\end{array}\right)\left(\begin{array}{c}
\Delta \Delta \boldsymbol{v} \\
\Delta \Delta \boldsymbol{p}
\end{array}\right)=\left(\begin{array}{c}
\boldsymbol{f}_{k}^{t+\Delta t} \\
\mathbf{0}
\end{array}\right)
$$

being the updating given by

$$
\begin{aligned}
& \boldsymbol{v}_{k+1}^{t+\Delta t}=\boldsymbol{v}_{k}^{t+\Delta t}+\Delta \Delta \boldsymbol{v}, \\
& p_{k+1}^{t+\Delta t}=p_{k}^{t+\Delta t}+\Delta \Delta p .
\end{aligned}
$$

Obviously, Eqs. (29) and (30) will be solved iteratively until convergence.

\subsection{Mixed natural neighbour approximations}

The above Eqs. (29) and (30) represent the linearised form of a Hellinger-Reissner-like functional. There are two essential variables, namely velocities and pressure. It is well known that trial spaces for these two variables must be chosen carefully, in order to avoid locking in the solution. In other words, these approximation spaces must be chosen so as to guarantee stability of the resulting formulation. This choice must thus verify the so-called inf-sup or Babuška-Brezzi condition $[2,5]$, together with the ellipticity condition.

The LBB condition may be written as

$$
\inf _{p^{h} \in \mathscr{P}_{h}} \sup _{\boldsymbol{v}^{h} \in \mathscr{U}_{h}} \frac{\int_{\Omega} p^{h} \boldsymbol{\nabla} \cdot \boldsymbol{v}^{h} \mathrm{~d} \Omega}{\left\|p^{h}\right\|_{0}\left\|\boldsymbol{v}^{h}\right\|_{1}}=\gamma_{h} \geqslant \gamma>0,
$$

where $\gamma$ is a positive constant independent of the mesh size, $h . \mathscr{U}_{h}$ and $\mathscr{P}_{h}$ represent, respectively, the displacement and pressure approximation spaces whose norms are defined as

$$
\begin{aligned}
& \|\cdot\|_{0}^{2}=\int_{\Omega}(\cdot)^{2} \mathrm{~d} \Omega \\
& \|\cdot\|_{1}^{2}=\int_{\Omega} \sum_{i, j=1}^{2}\left(\frac{\partial(\cdot)_{i}}{\partial x_{j}}\right)^{2} \mathrm{~d} \Omega .
\end{aligned}
$$

This condition is rarely proved analytically. Instead, its fulfillment is usually checked numerically.

In [15], an analysis of the stability of different mixed approximations employing natural neighbour-based interpolants was made. Although Sibson-Thiessen interpolation for velocities and pressures, respectively, has been found not to verify the inf-sup condition, it has rendered excellent results in previous works in the field of Fluid Mechanics [22,21] and rarely presents locking. In [15] an enriched formulation was developed by employing the partition of unity paradigm. Thus, a formulation with quadratic consistency in velocities and constant or linear pressures was found to verify the inf-sup condition. In this work, a Sibson-Thiessen formulation has been employed, which has one nodal degree of freedom less than the previous one.

\subsection{Theory of $\alpha$-shapes}

In free-surface flows like extrusion, and notably in three-dimensional cases, tracking the flow boundaries is a complex issue. Nodes on the boundary must be marked and checks must be done so as to verify the development of holes in the fluid or the coalescence of two or more flows. In this work we have employed a distinct technique, based in the extraction of the shape of the domain at each time step, without any supplementary information.

The concept of "shape" has usually no formal meaning in geometry (as opposed to others like diameter...). Edelsbrunner [12] gave a rigorous parameterisation of the set of shapes that a set of nodes can describe. To this end, a parameter $\alpha$, related to the level of detail up to which the set is to be represented, must be introduced.

An $\alpha$-shape is a polytope that is not necessarily convex nor connected, being triangulated by a subset of the Delaunay triangulation of the points. Thus, the empty circumcircle criterion holds. Let $N$ be a finite set of points in $\mathbb{R}^{3}$ and $\alpha$ a real number, with $0 \leqslant \alpha<\infty$. A $k$-simplex $\sigma_{T}$ with $0 \leqslant k \leqslant 3$ is defined as the convex hull of a subset $T \subseteq N$ of size $|T|=k+1$. Let $b$ be an $\alpha$-ball, that is, an open ball of radius $\alpha$. A $k$-simplex $\sigma_{T}$ is said to be $\alpha$-exposed if there exist an empty $\alpha$-ball $b$ with $T=\partial b \cap N$ where $\partial$ means the boundary of the ball. In other words, a $k$-simplex is said to be $\alpha$-exposed if an $\alpha$-ball that passes through its defining points contains no other point of the set $N$.

Thus, we can define the family of sets $F_{k, \alpha}$ as the sets of $\alpha$-exposed $k$-simplices for the given set $N$. This allows us to define an $\alpha$-shape of the set $N$ as the polytope whose boundary consists on the triangles in $F_{2, \alpha}$, the edges in $F_{1, \alpha}$ and the vertices or nodes in $F_{0, \alpha}$. 
A three-dimensional simplicial complex is a collection, $\mathscr{C}$, of closed $k$-simplices $(0 \leqslant k \leqslant 3)$ that satisfies:

(i) If $\sigma_{T} \in \mathscr{C}$ then $\sigma_{T^{\prime}} \in \mathscr{C}$ for every $T^{\prime} \subseteq T$.

(ii) The intersection of two simplexes in $\mathscr{C}$ is empty or is a face of both.

Each $k$-simplex $\sigma_{T}$ included in the Delaunay triangulation, $\mathscr{D}$, defines an open ball $b_{T}$ whose bounding spherical surface (in the general case) $\partial b_{T}$ passes through the $k+1$ points of the simplex. Let $\varrho_{T}$ be the radius of that bounding sphere, then, the family $G_{k, \alpha}$, is formed by all the $k$-simplexes $\sigma_{T} \in \mathscr{D}$ whose ball $b_{T}$ is empty and $\varrho_{T}<\alpha$. The family $G_{k, \alpha}$ does not necessarily form simplicial complexes, so Edelsbrunner and Mücke [12] defined the $\alpha$-complex, $\mathscr{C}_{\alpha}$, as the simplicial complex whose $k$-simplexes are either in $G_{k, \alpha}$, or else they bound $(k+1)$-simplexes of $C_{\alpha}$. If we define the underlying space of $\mathscr{C}_{\alpha},\left|\mathscr{C}_{\alpha}\right|$, as the union of all simplexes in $\mathscr{C}_{\alpha}$, the following relationship between $\alpha$-shapes and $\alpha$-complexes is found:

$$
\mathscr{S}_{\alpha}=\left|\mathscr{C}_{\alpha}\right| \quad \forall 0 \leqslant \alpha<\infty .
$$

In order to clarify the before presented concepts, consider some examples of $\alpha$-shapes computed from a cloud of points corresponding to the simulation of two-dimensional extrusion process. We restrict ourselves to geometrical concepts only.

Consider the extrusion example shown in Fig. 6, where the contour plot of equivalent plastic strain rate is depicted. The key idea of the method here proposed is to extract the shape of the domain at each time step by invoking the concept of $\alpha$ shape of the cloud. The $\alpha$ parameter will be obtained by geometrical considerations. In this case the radius at the inlet of the die, for instance, seems to be the smallest level of detail up to which the domain (i.e., the billet) must be represented. In order to appropriately represent this value, the nodal distance $h$ must be accordingly chosen.

In Fig. 7 some members of the family of $\alpha$-shapes of the cloud of points in its final configuration (corresponding to Fig. 6(b)) are depicted. In Fig. 7(a) the member for $\alpha=0$, i.e., the cloud of points itself, is shown. Note how, as $\alpha$ is increased, the number and size of the simplexes (in this case, triangles) that belong to the shape is increasing. For $\alpha=1.0$ we obtain an appropriate shape for the cloud. Note, however, that this is not an exact value to be determined at each time step. Since the number of $\alpha$-shapes is finite, there generally exists an interval of valid $\alpha$ values for a single shape. Finally, by increasing the $\alpha$ value, we arrive to the convex hull of the cloud of points (Fig. 7(f)).

Note, however, that the idea is not to choose a parameter $\alpha$ appropriate for the cloud. $\alpha$ represents a measure of the level of detail of the model. Once $\alpha$ is fixed by the user, a nodal cloud of typical size $h<\alpha$ must be generated in order to be able to reproduce the desired level of detail. Thus, $\alpha$ can be kept fixed throughout the simulation, although it can take different values at different regions of the model.

The use of $\alpha$-shapes has also a direct outcome in the imposition of essential boundary conditions. As mentioned before, if natural neighbour (Sibson or Laplace) interpolants are computed over an $\alpha$-shape of the cloud, linear interpolation of essential boundary conditions on convex as well as non-convex boundaries is guaranteed (see [8] and [7] for an in-deep demonstration of this property). We have coined this method the $\alpha$-shape based NEM, or simply $\alpha$-NEM [8].

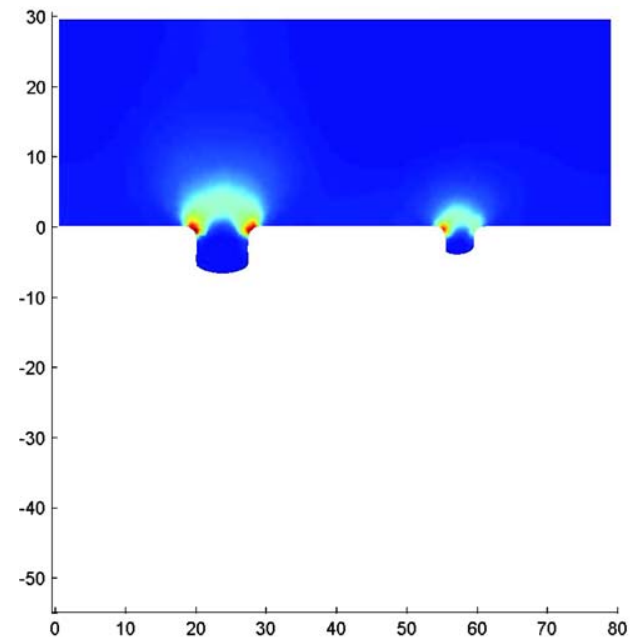

(a)
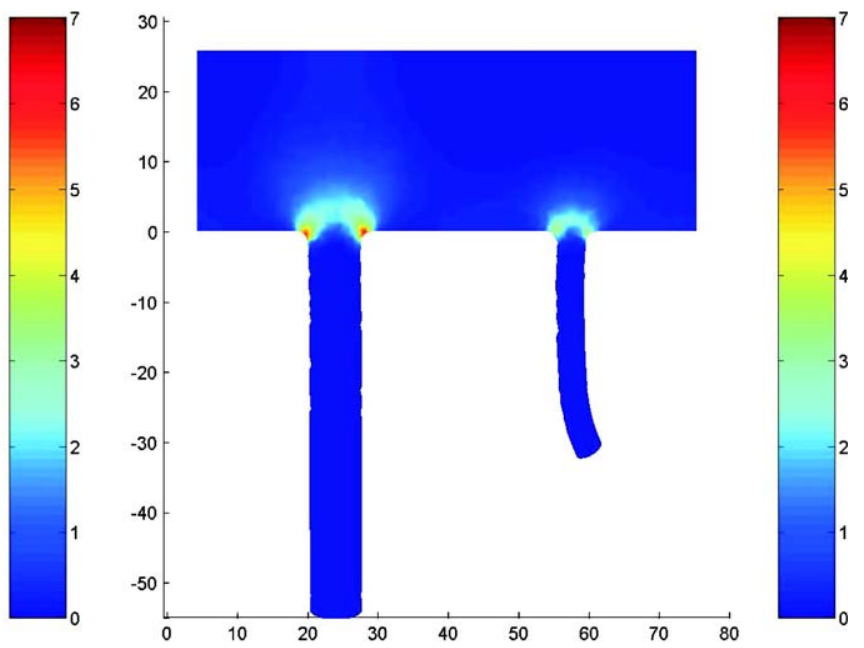

(b)

Fig. 6. Two snapshots of a two-dimensional simulation of an extrusion process. Equivalent plastic strain rate is depicted. 

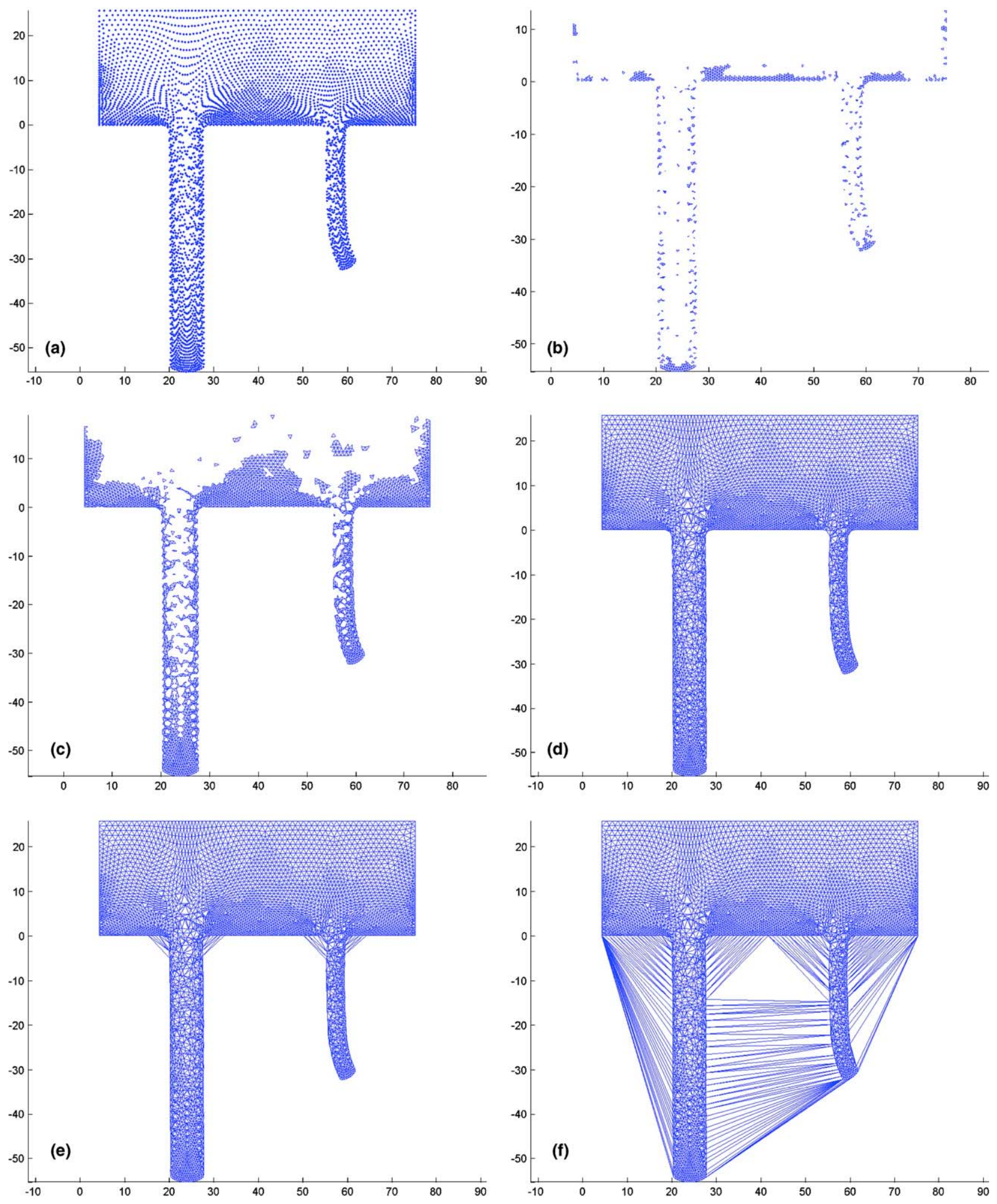

Fig. 7. Some members of the family of $\alpha$-shapes of the cloud of points used in the extrusion example. (a) $\mathscr{S}_{0}$ (the cloud of points), (b) $\mathscr{S}_{0.3}$, (c) $\mathscr{S}_{0.5}$, (d) $\mathscr{S}_{1.0}$, (e) $\mathscr{S}_{1.5}$ and (f) $\mathscr{S}_{\infty}$ (the convex hull of the set).

\subsection{Uncoupled thermo-mechanical procedure}

The resulting, coupled, non-linear problem is solved using a semi-implicit scheme combined with a fixed point iteration algorithm to treat the non-linear coupling. Moreover, the fixed point algorithm allows to de-couple the resolution of the mechanical and thermal problems at each iteration. Thus, assuming known the velocities and temperatures at time $t_{n}, v^{n}$ and $T^{n}$ respectively, we proceed as follows: 
(1) Explicit updating of the material domain. this process is performed moving all the nodes with the associated velocity: $\boldsymbol{x}_{I}^{n+1}=\boldsymbol{x}_{I}^{n}+\boldsymbol{v}_{I}^{n} \Delta t$.

(2) Velocity updating. Compute $\boldsymbol{v}_{0}^{n+1}$ using Eq. (29) and (30) assuming the temperature $T^{n}$ unchanged (the temperature field affects the pseudo-viscosity parameter $\mu$ (Eq. (13)) through the expression of the yield stress (Eq. (7)).

(3) Temperature updating. Update $T_{0}^{n+1}$ solving Eq. (24). The material derivative of the temperature field is discretised in a Lagrangian form, i.e.,

$\left.\frac{\mathrm{d} T}{\mathrm{~d} t}\right|_{I}=\frac{T\left(\boldsymbol{x}_{I}^{n+1}\right)-T\left(\boldsymbol{x}_{I}^{n}\right)}{\Delta t}$.

The resulting thermal diffusion problem is integrated by a semi-implicit Cranck-Nicolson scheme. In each step, the velocity field that defines the rate of heat generation (Eq. (25)) is assumed unchanged and given by the just computed kinematics $\boldsymbol{v}_{0}^{n+1}$.

(4) Velocity correction. We recompute step (2) assuming $T_{0}^{n+1}$ in the yield stress expression, leading to the new velocity field $\boldsymbol{v}_{1}^{n+1}$.

(5) Temperature correction. We recompute step (3) using $v_{1}^{n+1}$ in the rate of generation expression, leading to the field $T_{1}^{n+1}$.

(6) Convergence check.

if $\quad\left\|v_{1}^{n+1}-v_{0}^{n+1}\right\|<\varepsilon$

and

$\left|T_{1}^{n+1}-T_{0}^{n+1}\right|<\varepsilon$

then proceed to the next time step. Otherwise return to (4).

Remark 1. Using a fully explicit time discretisation the model becomes uncoupled at each time step.

Remark 2. If, after convergence in step (6), the previous nodal updating is recomputed, that is, if we return to step (1), then the fixed point algorithm is applied to solve the fully implicit coupled model.

This algorithm has proven to converge in at most three iterations per time step in the examples presented in Section 5.

\section{Numerical results}

\section{1. $3 D$ simulation of plane strain extrusion}

In this section we study a simple, academic, example of extrusion of a hollow, rectangular-shaped profile, where the dimension of one of the rectangle sides is much bigger than the other. Its geometry is shown in Fig. 8. Thus, the central section of the profile with respect to the largest rectangle side can be simulated under the assumption of plane strain, if we focus our interest in the region closer to the axis of symmetry. This section is labelled as "A" in Fig. 8. In addition, the other symmetry on section A has been taken into account in order to simulate only one half of the domain. Moreover,

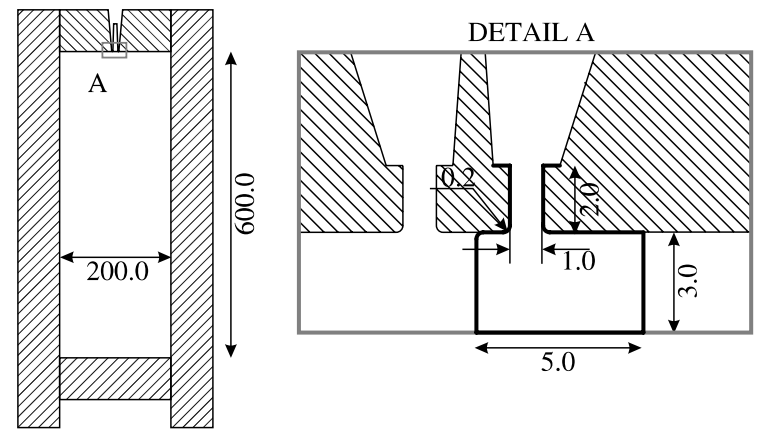

Fig. 8. Geometry of the extrusion die and detail of the simulated region. 


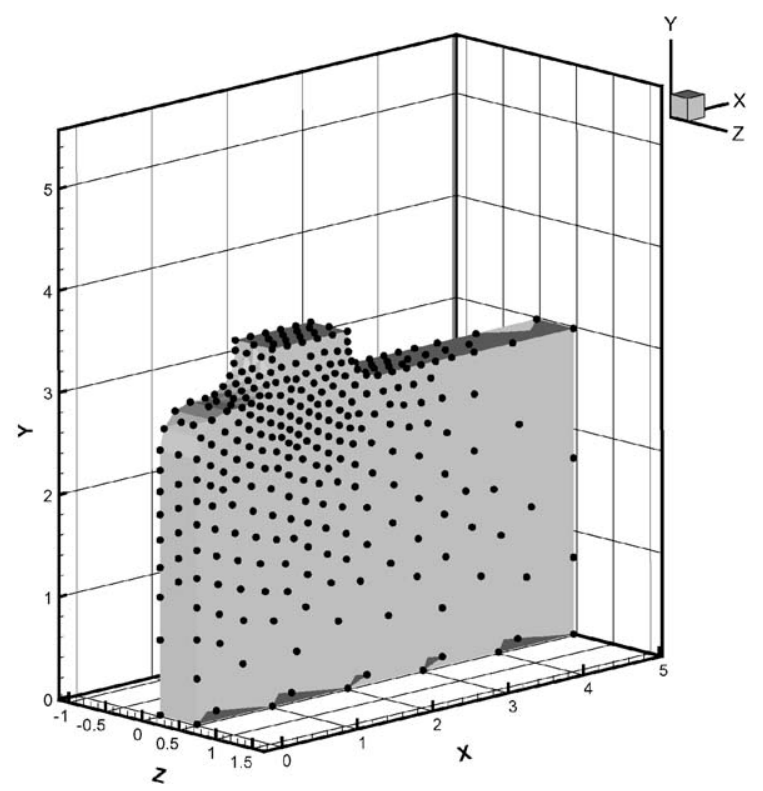

Fig. 9. Initial configuration of the cloud of points for the plane strain problem.

only the small region depicted in the detail of Fig. 8 has been simulated (this is a usual practice in the simulation of this kind of processes).

The cloud of points consisted of 575 nodes, whose initial configuration is shown in Fig. 9. Plane strain conditions were imposed by appropriate boundary conditions. The billet was assumed to be $0.5 \mathrm{~mm}$ thick because we conduct a 3D simulation, so we need to give an arbitrary value to the thickness in the out plane direction. A prescribed velocity of $1 \mathrm{~mm} / \mathrm{s}$, normal to the surface, was imposed at the lower and rightmost faces of the billet. Slip boundary conditions were assumed at the billet-die contact, where a null heat flux was imposed. Convective boundary conditions were assumed when the extruded billet gets out of the die, namely, at $y=4$, being the initial temperature $773 \mathrm{~K}$. Finally, in Table 2, thermal properties of aluminium are summarised.

The simulation ran for 96 time steps of $0.0025 \mathrm{~s}$. $\alpha$-shapes for several time steps are depicted in Fig. 10. Note that in this case the level of detail is bounded by the minimum radius of curvature to be reproduced, namely $0.2 \mathrm{~mm}$ (see detail in Fig. 8). The computed equivalent strain rate is depicted in Fig. 11. The corresponding temperature distributions for these time steps are shown in Fig. 12.

These results are in perfect agreement with those obtained in [1] using a two-dimensional formulation. Despite of the highly distorted tetrahedra (see Fig. 10), our results are also in good agreement with those obtained by Lof $[18,19]$ using an ALE formulation in a similar geometry. In our case, however, due to the updated Lagrangian character of the formulation, no special treatment was necessary to deal with free surfaces and the advection term in the heat equation, as required in ALE formulations (see [19] and references therein), where it is frequent that a flow out of the mesh appears when the extrudate gets out of the die. In this situation, mesh adjustments must be done.

\section{2. $3 D$ extrusion simulation}

In this section a more realistic simulation is presented. We deal with the extrusion of a cross-shaped profile whose geometry is shown in Fig. 13 and which was simulated using finite elements by Zhou et al. in [30]. The formulation described in Sections 2 and 4 has been employed, namely a Sellars-Tegart formulation for the behaviour of hot aluminium, as well as

Table 2

Thermal parameters for AA6063 aluminium alloy

\begin{tabular}{llr}
\hline Parameter & Units & Value \\
\hline$\beta$ & & 0.9 \\
$k$ & $\mathrm{~N} / \mathrm{s} \mathrm{K}$ & 180.0 \\
$\rho c_{p}$ & $\mathrm{~N} / \mathrm{mm}^{2} \mathrm{~K}$ & 2.39 \\
$h$ & $\mathrm{~N} / \mathrm{s} \mathrm{mm} \mathrm{K}$ & 0.1 \\
\hline
\end{tabular}



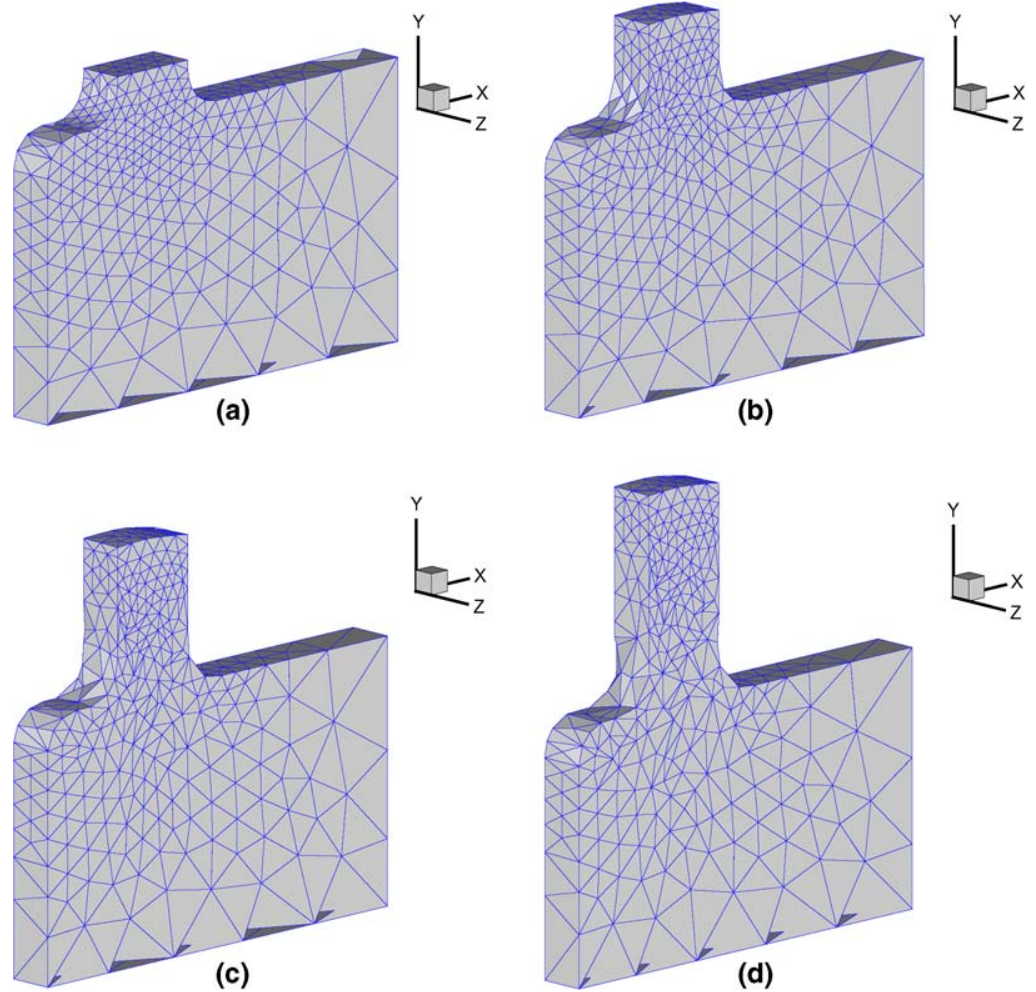

Fig. 10. $\alpha$-shapes at different time steps: (a) 1st step, (b) 30th step, (c) 60th step, (d) 96th step.
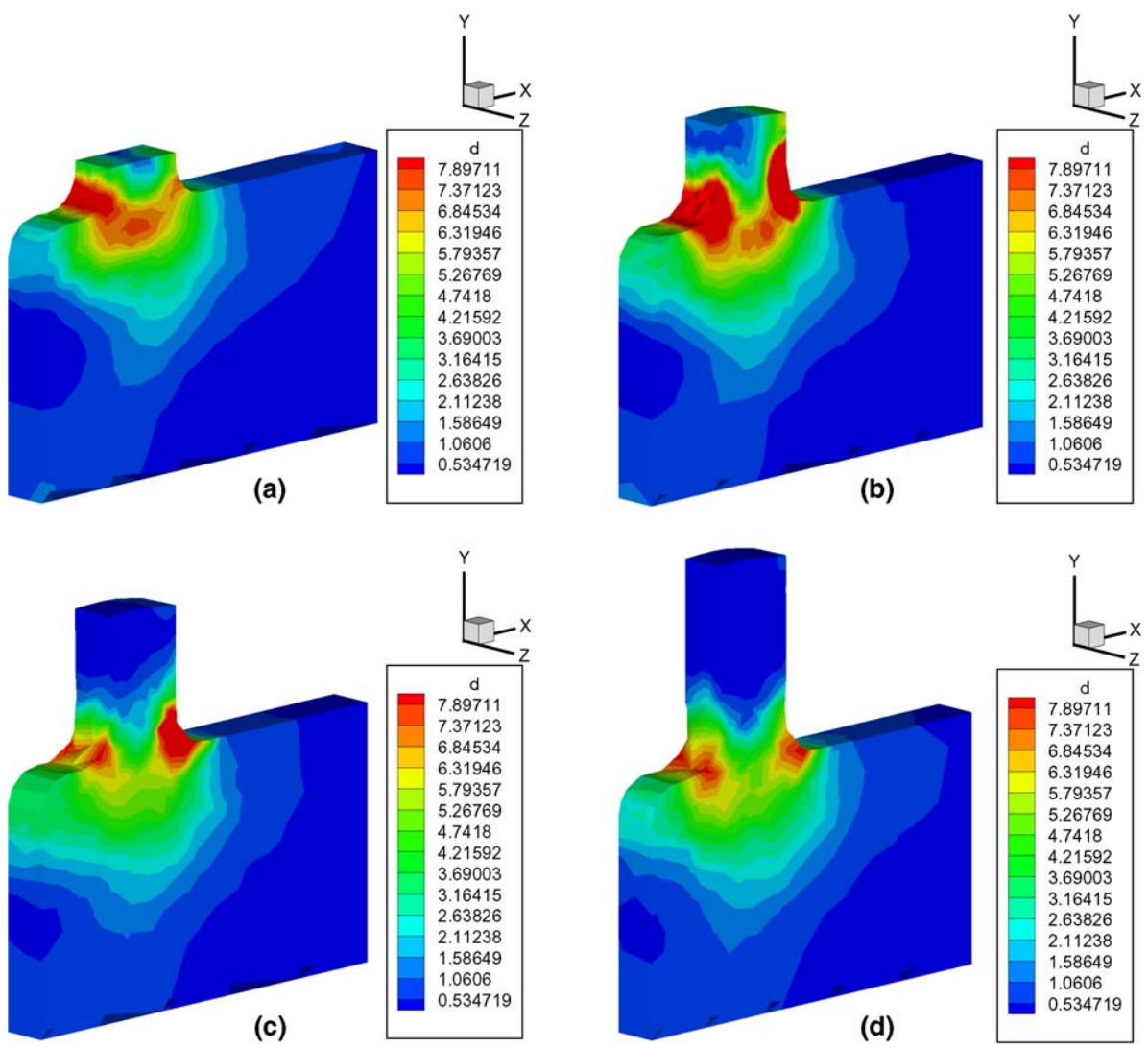

Fig. 11. Equivalent strain rate at different time steps: (a) 1st step, (b) 30th step, (c) 60th step, (d) 96th step. 

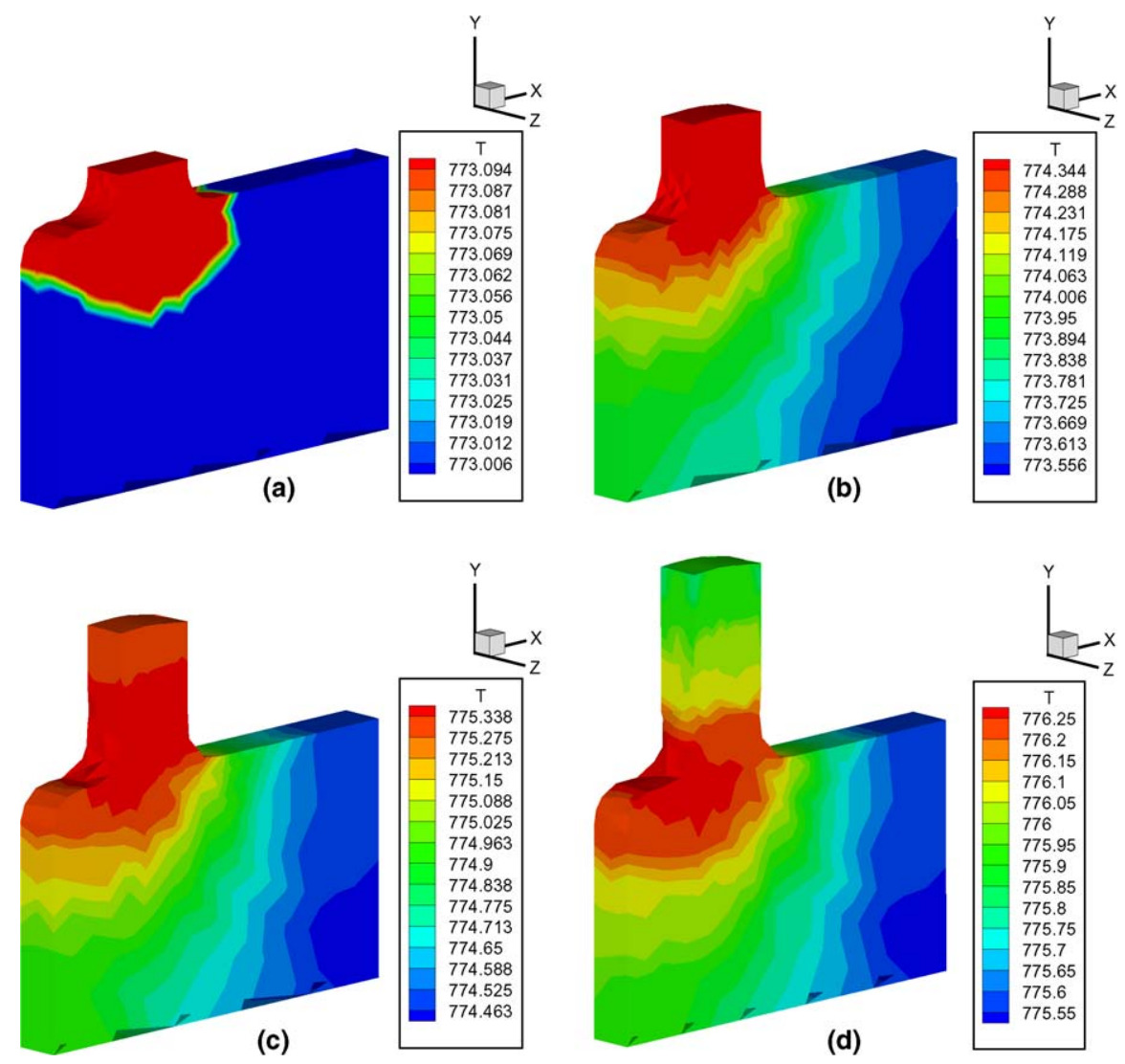

Fig. 12. Temperature distribution at different time steps: (a) 1st step, (b) 30th step, (c) 60th step, (d) 96 th step. Note the different temperature scales in each figure.

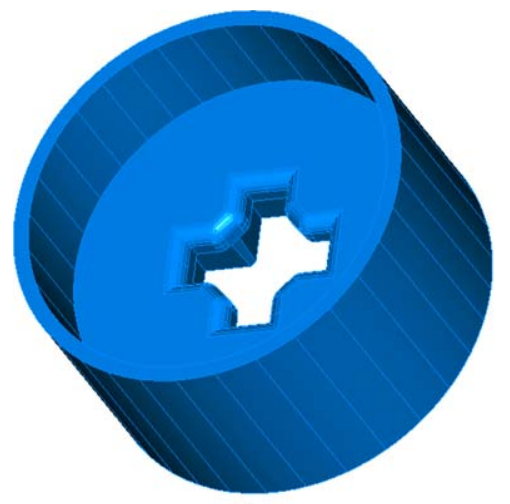

(a)

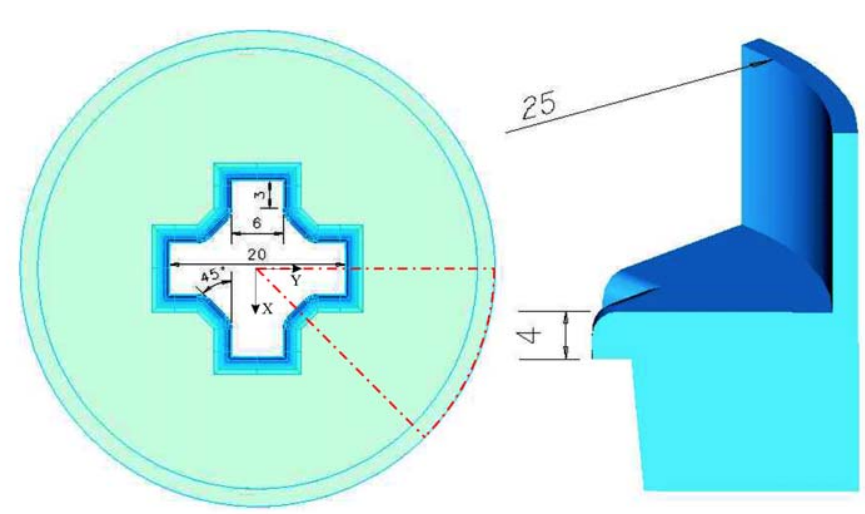

(b)

Fig. 13. Geometry of the die for the extrusion of the cross-shaped profile. (a) Perspective view and (b) geometry.

the semi-implicit algorithm previously described for solving the non-linear coupled model. Aluminium characteristics remain the same as in the previous example. An initial temperature of the whole billet of $723 \mathrm{~K}$ was considered, and slipping boundary conditions with the die walls were assumed, where the heat flux is supposed vanishing.

By applying appropriate boundary conditions only one eighth of the geometry was modelled. An initial cloud of 1313 nodes was considered as shown in Fig. 14. Extrusion was forced by imposing a velocity of $2 \mathrm{~mm} / \mathrm{s}$ at the outer face of the billet. The simulation ran in 42 time steps of $0.025 \mathrm{~s}$.

Resulting $\alpha$-shapes for time steps 1, 20, 30 and 42 are depicted in Fig. 15, being the equivalent strain rate and the temperature shown in Figs. 16 and 17 respectively. Note that, for post-processing purposes only, symmetry has been applied to the results in order to render the whole geometry of the extrudate. In Fig. 18 the interior distribution of temperatures is shown. 


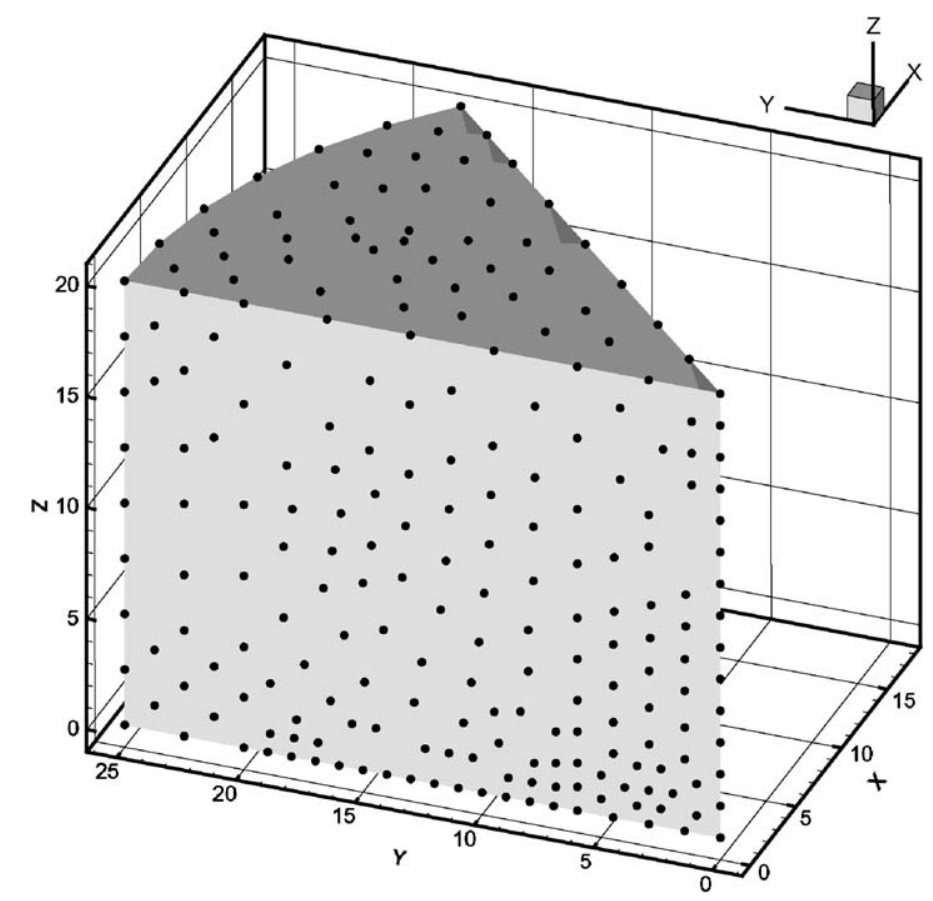

Fig. 14. Initial configuration of the cloud of points for the 3D problem.

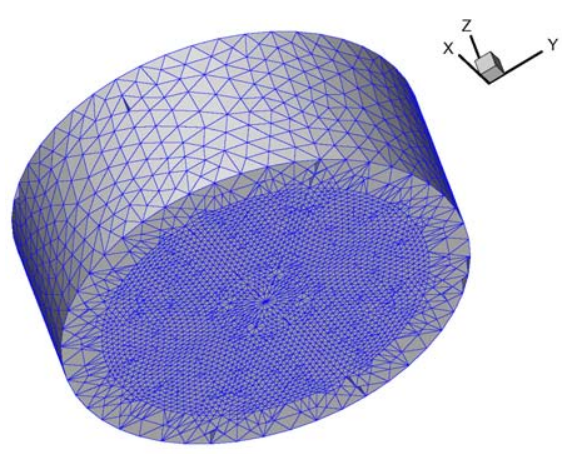

(a)

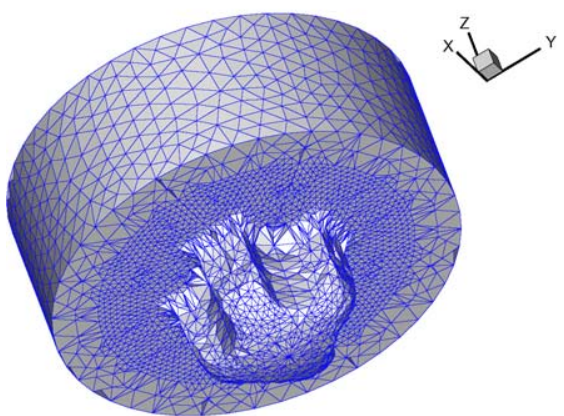

(c)

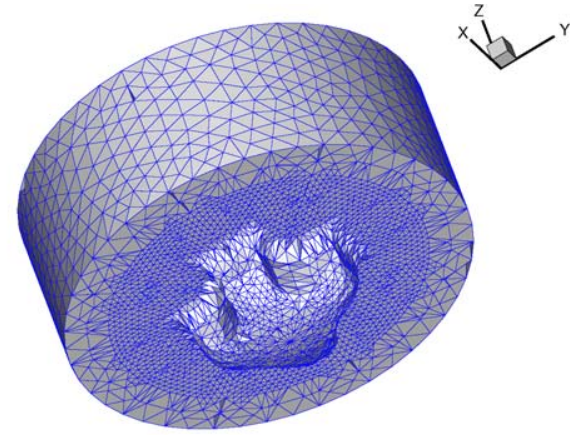

(b)

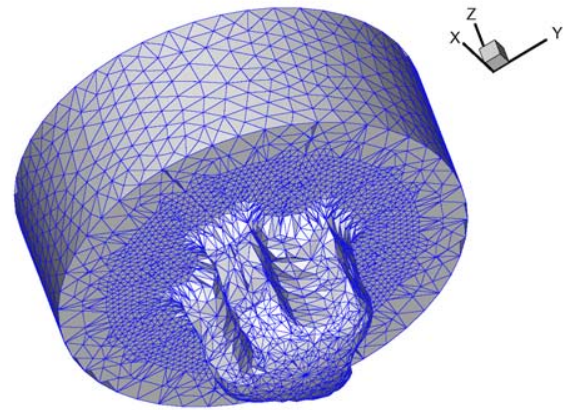

(d)

Fig. 15. $\alpha$-shapes for the extruded profile at different time steps: (a) 1st step, (b) 20th step, (c) 30th step, (d) 42nd step.

The updated Lagrangian formulation employed allowed us to predict the final shape of the profile with great accuracy. Results of this simulation can be compared to some extent (boundary conditions are not exactly the same) to those of Zhou et al. [30] by employing a FE updated Lagrangian approach and continuous remeshing. While temperature and equivalent strain rate distributions show similar patterns, it is expected that the lack of remeshing in our technique would produce less numerical diffusion in the results. Of course, this assert must be verified in additional test problems. 


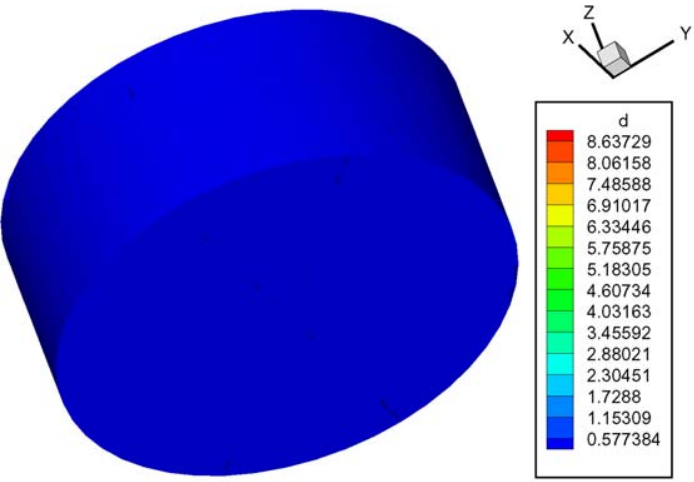

(a)

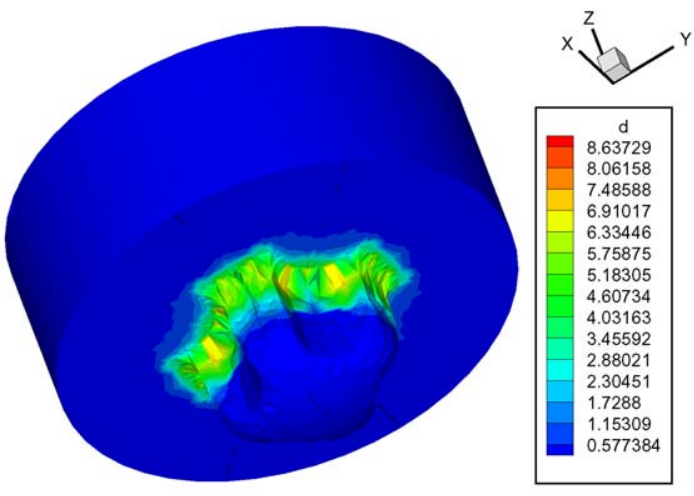

(c)

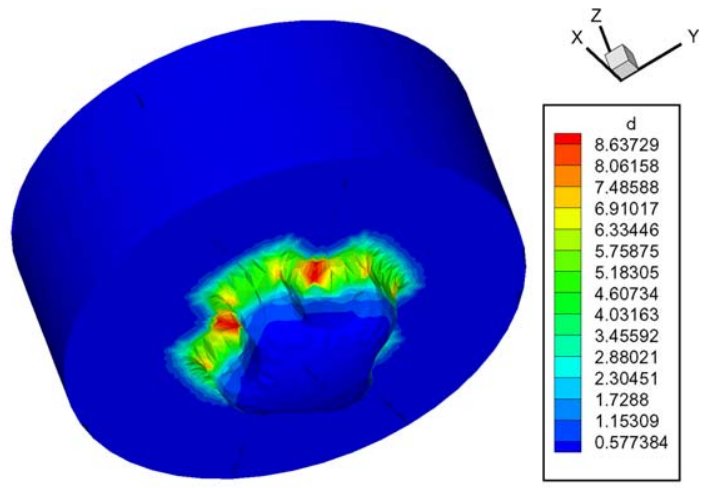

(b)

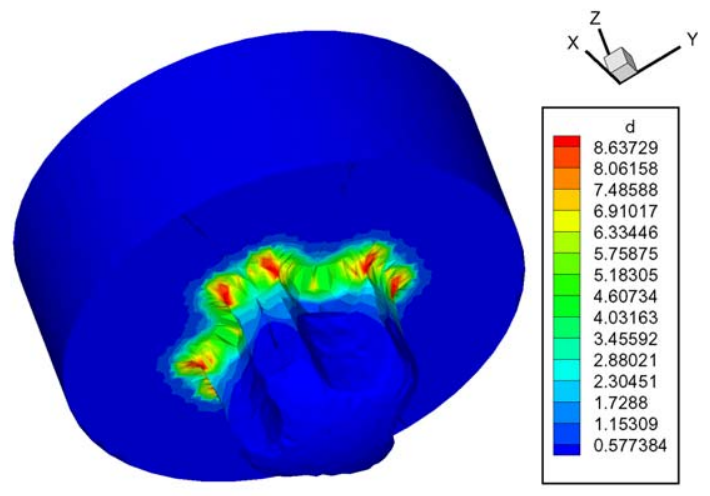

(d)

Fig. 16. Equivalent strain rate for different time steps: (a) 1st step, (b) 20th step, (c) 30th step, (d) 42nd step.

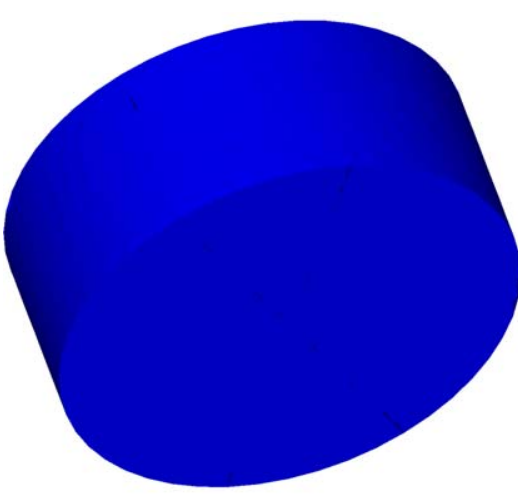

(a)

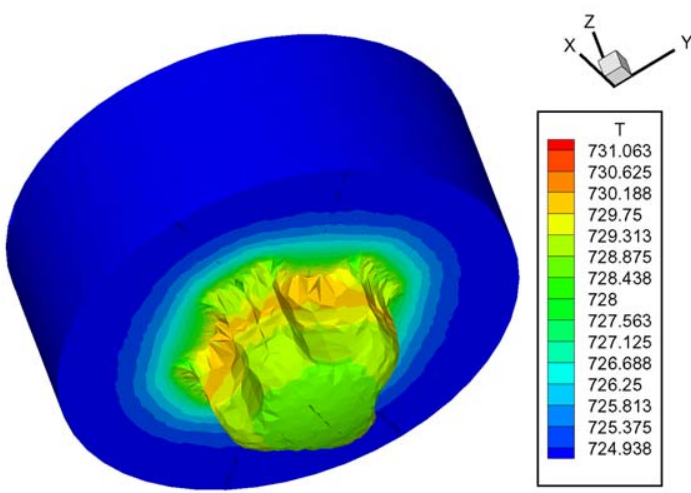

(c)

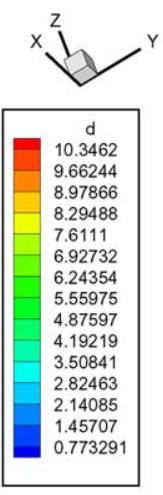

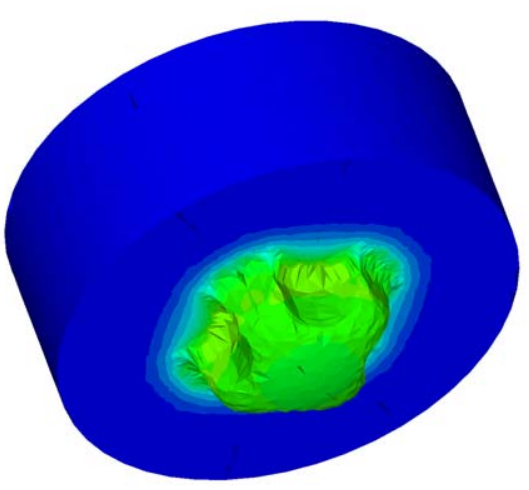

(b)

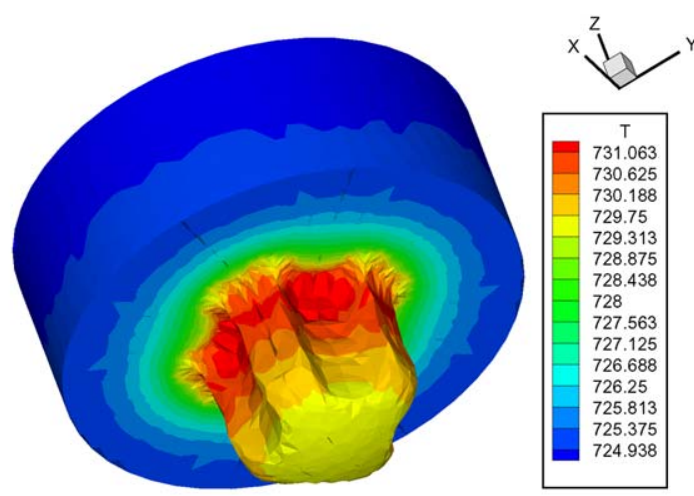

(d)

Fig. 17. Temperature profiles for different time steps: (a) 1st step, (b) 20th step, (c) 30th step, (d) 42nd step. 


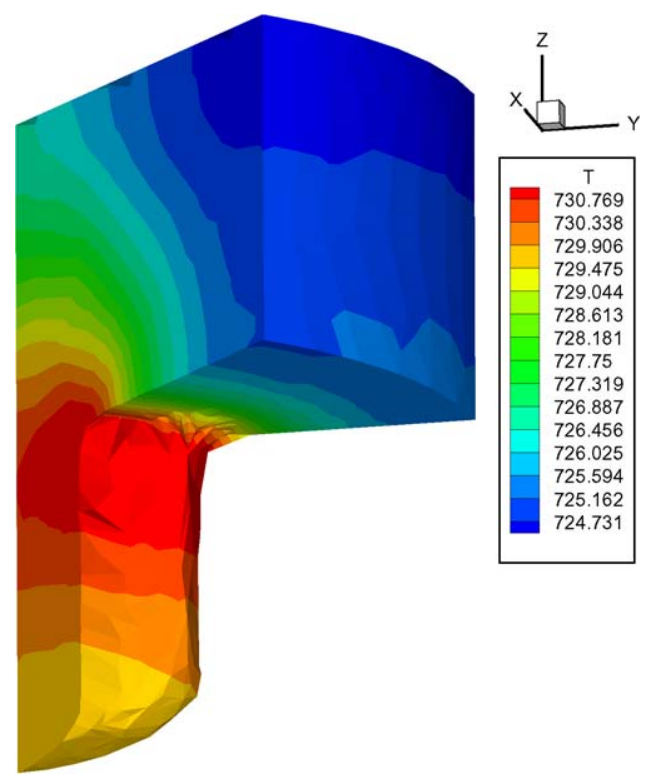

Fig. 18. Interior distribution of temperatures at the final time step.

\section{Conclusions}

In this paper we have presented a study of the potential applicability of the natural element method to the numerical simulation of aluminium extrusion. This technique allows us to employ an updated Lagrangian formulation in spite of the large material transformations that this process exhibits, avoiding remeshing. This means that the cloud of nodes remains the same throughout the simulations (though it is possible to add or delete nodes) and no mapping of secondary variables is necessary if stabilized nodal integration is used [14]. This Lagrangian approach also avoids many of the burden associated with ALE techniques, such as how to determine the velocity of the mesh.

We have employed a Sellars-Tegart formulation to model the behaviour of hot aluminium coupled with the appropriate thermal equations using a semi-implicit algorithm for solving the non-linear coupled mechanical model. From the geometrical point of view, an $\alpha$-shape-based approach to extract the geometry of the domain at each time step has been used. This avoids the use of complex geometrical information to locate nodes belonging to the boundary, such as those necessary to determine the presence or development of holes or merge of flows. As discussed, this has also a direct outcome in the imposition of essential boundary conditions, thus rendering a truly conforming method, in sharp contrast with the vast majority of meshless methods.

All this leaded us to conclude that the NEM has a big potential in its application to various forming processes (in general, those with large transformations) and in particular to extrusion processes. Moreover, the computational cost of the NEM, which is bigger than that of the FEM, is not a big difficulty in this class of problems, where the vast majority of time is passed in iterating in the Newton-Raphson scheme.

Other aspects remain, however, unclear, such as the more appropriate technique for the simulation of extrusion of thinwalled profiles. In this case, for instance, an $\alpha$-shape approach can be more demanding, since more nodes must be placed along the thickness of the resulting profile in order to accurately capture the geometry of the domain. Related approaches, such as the Constrained-NEM (C-NEM, [29]) could be probably applied in order to reduce the number of nodes employed in the simulation.

\section{References}

[1] I. Alfaro, J. Yvonnet, E. Cueto, F. Chinesta, M. Doblaré, Meshless methods with application to metal forming, Comput. Methods Appl. Mech. Engrg., in press.

[2] I. Babuška, The finite element method with Lagrange multipliers, Numer. Math. 20 (1973) 179-192.

[3] I. Babuška, J.M. Melenk, The partition of unity finite element method: basic theory and applications, Comput. Methods Appl. Mech. Engrg. 4 (1996) 289-314.

[4] T. Belytschko, Y.Y. Lu, L. Gu, Element-free Galerkin methods, Int. J. Numer. Methods Engrg. 37 (1994) $229-256$.

[5] F. Brezzi, On the existence, uniqueness and approximation of saddle-point problems arising from Lagrange multipliers, Revue Française d'Automatique Informatique Recherche Operationelle, Analyse Numérique 8 (1974) 129-151.

[6] J.-S. Chen, C.-T. Wu, S. Yoon, Y. You, A stabilized conforming nodal integration for Galerkin mesh-free methods, Int. J. Numer. Methods Engrg. 50 (2001) 435-466. 
[7] E. Cueto, J. Cegoñino, B. Calvo, M. Doblaré, On the imposition of essential boundary conditions in natural neighbour Galerkin methods, Commun. Numer. Methods Engrg. 19 (5) (2003) 361-376.

[8] E. Cueto, M. Doblaré, L. Gracia, Imposing essential boundary conditions in the natural element method by means of density-scaled $\alpha$-shapes, Int. J. Numer. Methods Engrg. 49-4 (2000) 519-546.

[9] E. Cueto, N. Sukumar, B. Calvo, M.A. Martínez, J. Cegoñino, M. Doblaré, Overview and recent advances in natural neighbour Galerkin methods, Arch. Comput. Methods Engrg. 10 (4) (2003) 307-384.

[10] B. Delaunay, Sur la Sphère Vide. A la memoire de Georges Voronoi, Izvestia Akademii Nauk SSSR Otdelenie Matematicheskii i Estestvennyka Nauk 7 (1934) 793-800.

[11] C.A.M. Duarte, J.T. Oden, An h-p adaptive method using clouds, Comput. Methods Appl. Mech. Engrg. 139 (1996) $237-262$.

[12] H. Edelsbrunner, E. Mücke, Three dimensional alpha shapes, ACM Trans. Graph. 13 (1994) 43-72.

[13] S. Fernández-Méndez, A. Huerta, Imposing essential boundary conditions in mesh-free methods, Comput. Methods Appl. Mech. Engrg. 193 (2004) $1257-1275$.

[14] D. González, E. Cueto, M.A. Martinez, M. Doblaré, Numerical integration in natural neighbour Galerkin methods, Int. J. Numer. Methods Engrg. 60 (12) (2004) 2077-2104.

[15] D. González, E. Cueto, M. Doblaré, Volumetric locking in natural neighbour Galerkin methods, Int. J. Numer. Methods Engrg. 61 (4) (2004) 611632.

[16] H. Hiyoshi, K. Sugihara, Two generalizations of an interpolant based on Voronoi diagrams, Int. J. Shape Model. 5 (2) (1999) $219-231$.

[17] S. Li, W.K. Liu, Meshfree and particle methods and their applications, Appl. Mech. Rev. 55 (2002) 1-34.

[18] J. Lof, Developments in finite element simulations of aluminium extrusion, Ph.D. thesis, Department of Mechanical Engineering, Applied Mechanics Section. University of Twente, P.O. Box 217, 7500 AE Enschede, The Netherlands, 2000.

[19] J. Lof, Elasto-viscoplastic FEM simulation of the aluminium flow in the bearing area for extrusion of thin-walled sections, J. Mater. Process. Technol. 114 (2001) 174-183.

[20] J. Lof, Y. Blokhuis, FEM simulations of the extrusion of complex thin-walled aluminium sections, J. Mater. Process. Technol. 122 (2002) $344-354$.

[21] M.A. Martinez, E. Cueto, I. Alfaro, M. Doblare, F. Chinesta, Updated Lagrangian free surface flow simulations with natural neighbour Galerkin methods, Int. J. Numer. Methods Engrg. 60 (13) (2004) 2105-2129.

[22] M.A. Martínez, E. Cueto, M. Doblaré, F. Chinesta, Fixed mesh and meshfree techniques in the numerical simulation of injection processes involving short fiber suspensions, J. Non-Newton. Fluid Mech. 115 (2003) 51-78.

[23] R. Sibson, A vector identity for the Dirichlet tesselation, Math. Proc. Cambridge Philos. Soc. 87 (1980) 151-155.

[24] R. Sibson, A brief description of natural neighbour interpolation, in: V. Barnett (Ed.), Interpreting Multivariate Data, John Wiley, 1981, pp. 21-36.

[25] N. Sukumar, B. Moran, T. Belytschko, The natural element method in solid mechanics, Int. J. Numer. Methods Engrg. 43 (5) (1998) $839-887$.

[26] N. Sukumar, B. Moran, A. Yu Semenov, V.V. Belikov, Natural neighbor Galerkin methods, Int. J. Numer. Methods Engrg. 50 (1) (2001) 1-27.

[27] A.H. Thiessen, Precipitation averages for large areas, Mon. Weather Rep. 39 (1911) 1082-1084.

[28] G.M. Voronoi, Nouvelles Applications des Paramètres Continus à la Théorie des Formes Quadratiques. Deuxième Memoire: Recherches sur les parallélloèdres Primitifs, J. Reine Angew. Math. 134 (1908) 198-287.

[29] J. Yvonnet, D. Ryckelynck, P. Lorong, F. Chinesta, A new extension of the Natural Element method for non-convex and discontinuous problems: the Constrained Natural Element method, Int. J. Numer. Methods Eng. 60 (8) (2004) 1452-1474.

[30] J. Zhou, L. Li, J. Duszczyk, 3D FEM simulation of the whole cycle of aluminium extrusion throughout the transient state and the steady state using the updated Lagrangian approach, J. Mater. Process. Technol. 134 (2003) 383-397.

[31] O.C. Zienkiewicz, P.N. Godbolet, Flow of plastic and visco-plastic solids with special reference to extrusion and forming processes, Int. J. Numer. Methods Engrg. 8 (1974) 3-16.

[32] O.C. Zienkiewicz, E. Oñate, J.C. Heinrich, Plastic flow in metal forming. (I) Coupled thermal (II) Thin sheet forming, In: Applications of Numerical Methods to Forming Processes, AMD-vol 28, pp. 107-120, 1978.

[33] O.C. Zienkiewicz, P.C. Pain, E. Oñate, Flow of solids during forming and extrusion: some aspects of numerical solutions, Int. J. Solids Struct. 14 (1978) 15-38. 